\title{
Scaling of the anomalous Hall current in $\mathrm{Fe}_{100-x}\left(\mathrm{SiO}_{2}\right)_{x}$ films
}

\author{
W. J. Xu, ${ }^{1}$ B. Zhang, ${ }^{2}$ Q. X. Wang, ${ }^{2}$ W. B. Mi, ${ }^{3}$ Z. Wang, ${ }^{1}$ W. Li,${ }^{1}$ R. H. Yu, ${ }^{4}$ and X. X. Zhang ${ }^{2, *}$ \\ ${ }^{1}$ Institute of Nanoscience and Technology, The Hong Kong University of Science and Technology (HKUST), \\ Clear Water Bay, Kowloon, Hong Kong, China \\ ${ }^{2}$ Thin Film Lab, 4700 King Abdullah University of Science and Technology (KAUST), Thuwal 23900-6900, Kingdom of Saudi Arabia \\ ${ }^{3}$ Tianjin Key Laboratory of Low Dimensional Materials Physics and Preparing Technology, Institute of Advanced Materials Physics, \\ Faculty of Science, Tianjin University, Tianjin 300072, China \\ ${ }^{4}$ School of Materials Science and Engineering, Beihang University, Beijing 100191, China \\ (Received 15 December 2010; revised manuscript received 16 March 2011; published 20 May 2011)
}

\begin{abstract}
To study the origin of the anomalous Hall effect, $\mathrm{Fe}_{100-x}\left(\mathrm{SiO}_{2}\right)_{x}$ granular films with a volume fraction of $\mathrm{SiO}_{2}$ $(0 \leqslant x \leqslant 40.51)$ were fabricated using cosputtering. Hall and longitudinal resistivities were measured in the temperature range of 5-350 $\mathrm{K}$ with magnetic fields up to $5 \mathrm{~T}$. As $x$ increased from 0 to 40.51 , the anomalous Hall resistivity and longitudinal resistivity increased by about four and three orders in magnitude, respectively. Analysis of the results revealed that the normalized anomalous Hall conductivity is a constant for all of the samples, which may suggest a scattering-independent anomalous Hall conductivity in Fe.
\end{abstract}

DOI: 10.1103/PhysRevB.83.205311

PACS number(s): 73.61.-r, 72.15.Gd, 75.70.-i, 81.15.Cd

\section{INTRODUCTION}

A magnetization $(M)$-dependent Hall resistivity, $R_{S} 4 \pi M$, is generally observed in magnetic materials and called the anomalous Hall effect (AHE),

$$
\rho_{x y}=R_{O} B+R_{S} 4 \pi M,
$$

where $\rho_{x y}$ and $R_{O} B$ are the total and ordinary Hall resistivities, respectively, and $B$ is the magnetic induction in the materials. ${ }^{1}$ Although AHE has been observed for more than a century, it remains quite poorly understood. Karplus and Luttinger ${ }^{2}$ first proved theoretically that an anomalous Hall conductivity (AHC) can be caused by spin-orbit coupling in Bloch bands in a perfect ferromagnetic crystal, a purely quantum-mechanical origin of AHE. AHE was then considered an intrinsic effect. Later, the extrinsic scattering mechanism of skew scattering (SS) was proposed by $\mathrm{Smit}^{3}$ to argue that the intrinsic AHE should vanish in a periodical lattice.

In the SS model, the spin-up and spin-down electrons are scattered in the opposite sides by impurities due to the spin-orbit coupling. The nonzero spin polarization (the difference in the density of states for spin-up and spin-down electrons at the Fermi level) in ferromagnetic materials leads to the observed anomalous Hall resistivity $\left(\rho_{x y}^{A}\right)$. Since both the longitudinal and anomalous Hall resistivities originate from scatterings of impurities in this model, they should be correlated. The SS model indeed predicted a linear dependence of anomalous Hall resistivity on the longitudinal resistivity $\left(\rho_{x x}\right)$, i.e., $\rho_{x y}^{A} \sim \rho_{x x}$. This linear dependence was definitely observed in some materials. However, in most experiments an approximately quadratic proportionality $\rho_{x y}^{A} \sim \rho_{x x}^{2}$ was observed. The side-jump (SJ) mechanism was then proposed by Berger ${ }^{4}$ to interpret the quadratic dependence. In the SJ model, the trajectories of scattered electrons shift to different sides depending on their spins due to the spin-orbit coupling. Again, the imbalance of spin-up and spin-down electrons in ferromagnetic materials results in the AHE. The SJ mechanism indeed predicted a quadratic dependence.

Since linear and/or quadratic dependence of AHE on longitudinal resistivity has been commonly observed exper- imentally, it has been suggested that AHE in ferromagnetic materials is an extrinsic effect and is due solely to carrier scatterings by impurities. However, it has not been possible to compare the theories and experiments quantitatively because it is very difficult to model the scattering potentials in real materials. ${ }^{5,6}$

Only recently, the intrinsic mechanism proposed by Karplus and Luttinger was re-examined. ${ }^{6-10}$ It was found that the anomalous Hall conductivity can indeed be caused by the Berry curvatures in momentum space, ${ }^{6-10}$ where the Berry curvature is defined by $\Omega_{n}(\mathrm{k})=-\operatorname{Im}\left\langle\nabla_{\mathrm{k}} u_{n \mathrm{k}}|\times| \nabla_{\mathrm{k}} u_{n \mathrm{k}}\right\rangle$, where $u_{n \mathrm{k}}$ is the Bloch wave function in the $n$th band. The anomalous Hall conductivity can then be written as the sum of the Berry curvatures over the occupied Bloch states. Intrinsic anomalous Hall conductivity has been evaluated numerically in body-centered-cubic iron, ${ }^{6}$ ferromagnetic semiconductors, ${ }^{7}$ and oxides. ${ }^{9}$ It was found that the intrinsic anomalous Hall conductivity can be quite large, and a quantitative agreement between theory and experiment is now possible.

The anomalous Hall effect in magnetic granular films was studied by different groups ${ }^{11-13}$ in the 1990s. Pakhomov et al. observed the giant anomalous Hall effect in $\mathrm{Ni}_{x}\left(\mathrm{SiO}_{2}\right)_{100-x}$, where AHE increased nearly four orders in magnitude as $x$ decreased from 100 to 53 or in the vicinity of the percolation threshold. ${ }^{11}$ They found that anomalous Hall resistivity $\left(\rho_{x y}^{A}\right)$ as a function of longitudinal resistivity $\left(\rho_{x x}\right)$ can be fitted to a power law $\rho_{x y}^{A} \sim \rho_{x x}^{n}$, with $n \sim 0.7$ for the metal volume fraction higher than the percolation threshold. No mechanism was suggested to be responsible for giant AHE in this system. Yan and $\mathrm{Zhao}^{12}$ studied the Hall effect in the $\mathrm{Fe}_{x}\left(\mathrm{SiO}_{2}\right)_{100-x}$ granular films of $0.5 \mu \mathrm{m}$ thickness with $x=67,56,53,52$, and 50, i.e., the metal volume fraction above the percolation threshold. They found that both the ordinary Hall resistivity and the AHE resistivity increased about four orders in magnitude as $x$ decreased from 100 to 50 . The authors ascribed the giant AHE to the sharp reduction of carrier density near the percolation. Aronzon et al. studied the Hall effect in the hopping conduction regime in the $\mathrm{Fe}_{x}\left(\mathrm{SiO}_{2}\right)_{100-x}$ films with a volume fraction of Fe less than the percolation threshold $x_{c}$ $\left(\sim 60\right.$ in their study). ${ }^{13}$ They found a $\rho_{x y}^{A} \sim \rho_{x x}^{n}$ dependence 
with a much lower $n$ value, $0.44 \leqslant n \leqslant 0.6$ for samples with different metal volume fraction $x$. They attributed the low values of the exponent to the characteristic features of the Hall effect in the hopping regime-the interference of the amplitudes of tunneling transitions in a set of three granules.

A few years ago, Lee et al. studied the Hall effect in the ferromagnetic spinel $\mathrm{CuCr}_{2} \mathrm{Se}_{4}-x \mathrm{Br}_{x}$ with different $x(0 \leqslant$ $x \leqslant 1) .{ }^{14}$ By varying $x$ from 0 to 0.85 , the low-temperature longitudinal resistivity increased about 270 times, although all the samples were still metallic except for the sample $x=0.85$. For the $x=0.85$ sample, the resistivity increased as the temperature decreased. The resistivity increased more than 1000 times for $x=1$ sample in comparison with the $x=0$ sample. Meanwhile, the anomalous Hall resistivity changed more than three orders in magnitude. Interestingly, the low-temperature saturation magnetization was almost unchanged in those samples. The key finding in this study is the observation of the quadratic dependence of the normalized anomalous Hall resistivity (by the carrier density) on the longitudinal resistivity, i.e., $\rho_{x y}^{A} / n \propto \rho_{x x}^{2}$. Here, the anomalous Hall conductivity $\sigma_{\text {AHE }}$ was defined as $\rho_{x y}^{A} / \rho_{x x}^{2}$. Based on the simple theoretical model by Nozières and Lewiner (N-L model), ${ }^{15}$ $J_{x y}^{A}=2 n e^{2} \lambda E \times S$, the authors pointed out that this is the direct test of the dissipationless nature of Hall current, where $\lambda$ is the enhanced spin-orbit parameter, $S$ is the electron spin, $n$ is the carrier density, and $e$ is the electron charge. In the theory of Karplus and Luttinger, the Hall current $J_{x y}^{A}$ is dissipationless: $J_{x y}^{A}$ remains constant even as the longitudinal current $(\boldsymbol{J} / / E)$ is degraded by scattering from added impurities. ${ }^{14}$ The N-L model may be too simple for a complicated system such as $\mathrm{CuCr}_{2} \mathrm{Se}_{4-x} \mathrm{Br}_{x}$. With the increase of $\mathrm{Br}$ concentration, more and more conducting paths are cut off, although the magnetic properties are not significantly altered. ${ }^{14,16}$ Goodenough $^{17}$ interpreted the electrical conduction in $\mathrm{CuCr}_{2} \mathrm{Se}_{4}$ crystal as follows. The $\mathrm{Cu}^{2+}$ ion in $\mathrm{CuCr}_{2} \mathrm{Se}_{4}$ behaves as $\mathrm{Cu}^{1+}$ plus a hole, i.e., each $\mathrm{CuCr}_{2} \mathrm{Se}_{4}$ molecule contributes one hole. The electrical conduction can then be ascribed to the hopping of electrons between the holes (because $\mathrm{Cu}$ ions cannot move physically). When a Se atom is substituted by a $\mathrm{Br}$, the $\mathrm{Cu}$ atom becomes a $\mathrm{Cu}^{1+}$ ion in the $\mathrm{CuCr}_{2} \mathrm{Se}_{3} \mathrm{Br}$ molecule and the molecule no longer contributes any carrier to the electrical conduction. In this case, the electron hopping to the molecule $\mathrm{CuCr}_{2} \mathrm{Se}_{3} \mathrm{Br}$ is blocked, which may be considered a broken conducting path. The broken conduction path can certainly be considered a scattering center. As Br substitution increases, the electrical conduction (longitudinal resistivity) in $\mathrm{CuCr}_{2} \mathrm{Se}_{4-x} \mathrm{Br}_{x}$ might be described by the three-dimensional (3D) bond percolation model. ${ }^{18}$

It is well known that the metal-insulator granular materials also show the percolation behavior as the metal volume fraction decreases ${ }^{11,12,18-20}$ and may be described by the bond percolation model ${ }^{20}$ Therefore, it is interesting to explore if there is the same correlation $\rho_{x y}^{A} / n \propto \rho_{x x}^{2}$ in the ferromagnetic granular systems as observed in $\mathrm{CuCr}_{2} \mathrm{Se}_{4-x} \mathrm{Br}_{x}$.

In this study, $\mathrm{Fe}_{100-x}\left(\mathrm{SiO}_{2}\right)_{x}$ granular films were chosen because $\mathrm{Fe}$ is a simple ferromagnet and AHE in pure iron crystal has been demonstrated to be intrinsic by the numerical calculations. Although many models and theories have been proposed for AHE, ${ }^{21,22}$ no theories or models have been dedicated to the systems with strong disorders, such as the metal-insulator composites whose electrical transport properties should be described within the percolation theories. The work presented in this paper may lead to a deeper understanding of AHE in the strongly disordered systems.

\section{EXPERIMENTAL}

$\mathrm{Fe}_{100-x}\left(\mathrm{SiO}_{2}\right)_{x}$ granular films $\sim 300 \mathrm{~nm}$ thick with different $\mathrm{SiO}_{2}$ volume fractions were fabricated by cosputtering on the glass, quartz, and Kapton substrates. In this study, we focused on the samples with a metal volume fraction higher than the percolation threshold, i.e., $\mathrm{SiO}_{2}$ volume fraction $0 \leqslant x \leqslant$ 40.51. The base pressure and argon pressure for sputtering were $2 \times 10^{-7}$ Torr and $4 \times 10^{-3}$ Torr, respectively. During the deposition, the substrates were at room temperature. Different $\mathrm{SiO}_{2}$ volume fractions were obtained by controlling the relative sputtering powers applied to $\mathrm{Fe}$ and $\mathrm{SiO}_{2}$ targets and confirmed by x-ray fluorescence (XRF) measurements. The film thickness was controlled by the sputtering time, then measured by a Veeco-Dektak surface profiler. To perform Hall and longitudinal resistivity measurements simultaneously and to guarantee that the data of the Hall and longitudinal resistivity were obtained from the same sample, masks were used to fabricate patterned samples. Patterned films on glass substrates were used for the resistivity and Hall measurements with a Quantum Design physical property measurement system (PPMS). Samples deposited on quartz and Kapton substrates were used for magnetic measurements using a Quantum Design magnetic property measurement system (MPMS).

\section{RESULTS AND DISCUSSION}

The Hall resistivity $\rho_{x y}$ and magnetization for all the samples were measured with a magnetic field applied perpendicularly to the film plane in a range of $-5 \mathrm{~T} \leqslant H \leqslant 5 \mathrm{~T}$ and at temperatures ranging from 5 to $350 \mathrm{~K}$. Figure 1 shows the field dependence of the Hall resistivity and magnetization of
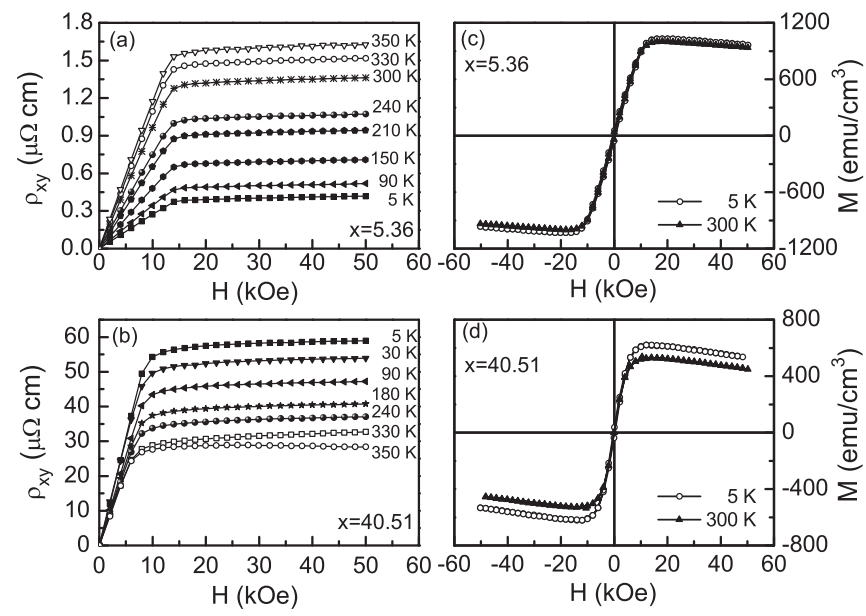

FIG. 1. Field-dependent Hall resistivity obtained at different temperatures for $\mathrm{Fe}_{100-x}\left(\mathrm{SiO}_{2}\right)_{x}$ films (a) $x=5.36$ and (b) $x=40.51$. Field-dependent magnetization measured at different temperatures for samples with different compositions: (c) $x=5.36$ and (d) $x=$ 40.51 . 
the $\mathrm{Fe}_{94.64}\left(\mathrm{SiO}_{2}\right)_{5.36}$ and $\mathrm{Fe}_{59.49}\left(\mathrm{SiO}_{2}\right)_{40.51}$ films at different temperatures. The Hall resistivities in Fig. 1 include both the ordinary Hall effect (OHE) and the AHE contributions [Eq. (1)].

As expected, the field-dependent Hall resistivity curves [Figs. 1(a) and 1(b)] have behaviors very similar to that of field-dependent magnetization curves [Figs. 1(c) and 1(d)]. The behavior of the magnetization curves is typical for a soft ferromagnetic film whose magnetization lies in the film plane when the field is applied perpendicularly to the film plane. As the applied field is increased, the magnetization is forced to rotate to the field direction gradually, which results in a linearly field-dependent magnetization until saturation at about 14 and $11 \mathrm{kOe}$ for $x=5.36$ and 40.51, respectively, as shown in Figs. 1(c) and 1(d). Due to the linear dependence of AHE on magnetization [Eq. (1)], a linear change in Hall resistivity is observed at low fields and saturation at high fields. No hysteretic behavior was observed for either sample.

Above the saturation field, the weak linear increase of Hall resistivity should be from the ordinary Hall effect, the first term in the right-hand side of Eq. (1), because the magnetization was saturated (the linear decrease in magnetization is due to the negative diamagnetic contribution from the substrate). By fitting this linear part of the Hall signal, we obtained the ordinary Hall coefficients $\left(R_{O}\right)$ that were used to calculate the effective carrier densities of the samples. From the fitting, the saturated AHE was also extracted. Figure 2 shows the saturated AHE obtained at $5 \mathrm{~K}$ for all the samples with different compositions. Overall, AHE increases as $x$ increases. The ratio of $\rho_{x y}^{A}(x=40.51) / \rho_{x y}^{A}(0)$ is about 14275 , an increase in magnitude of more than four orders. The giant increase in AHE is referred to as a giant Hall effect (GHE). ${ }^{11}$ We will see below that this giant change in AHE is essential to make a reliable scaling between AHE and the longitudinal resistivity.

Shown in Fig. 3(a) are the temperature-dependent longitudinal resistivity curves measured under zero magnetic field from 5 to $350 \mathrm{~K}$ for samples with various compositions. It is evident that the behaviors of the resistivity curves depend strongly on $x$. For pure Fe and samples with $x$ smaller than 30 , resistivity increased monotonically as temperature increased, a typical metallic behavior. At $x>30$, the resistivity decreased monotonically as temperature increased, a characteristic of semiconductors. In Fig. 3(b), we plotted the $300 \mathrm{~K}$ temperature coefficient of the resistivity (TCR), $\mathrm{d}(\ln \rho) / \mathrm{d}(\ln T)$, as a

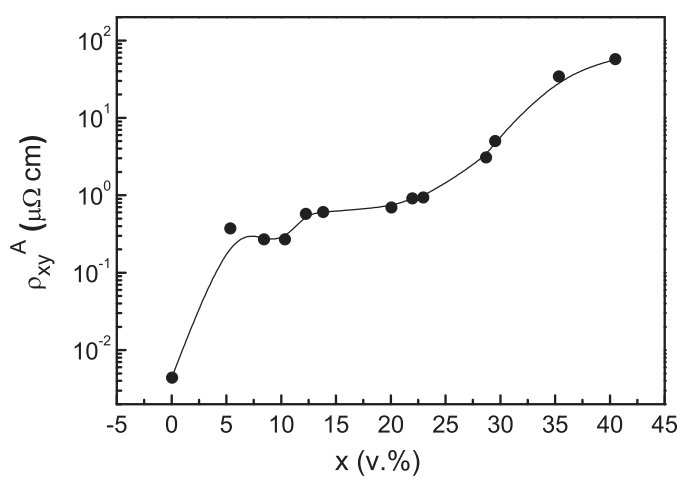

FIG. 2. Saturated anomalous Hall resistivity as a function of the $\mathrm{SiO}_{2}$ volume fraction at $5 \mathrm{~K}$. The solid line is a guide to the eye.
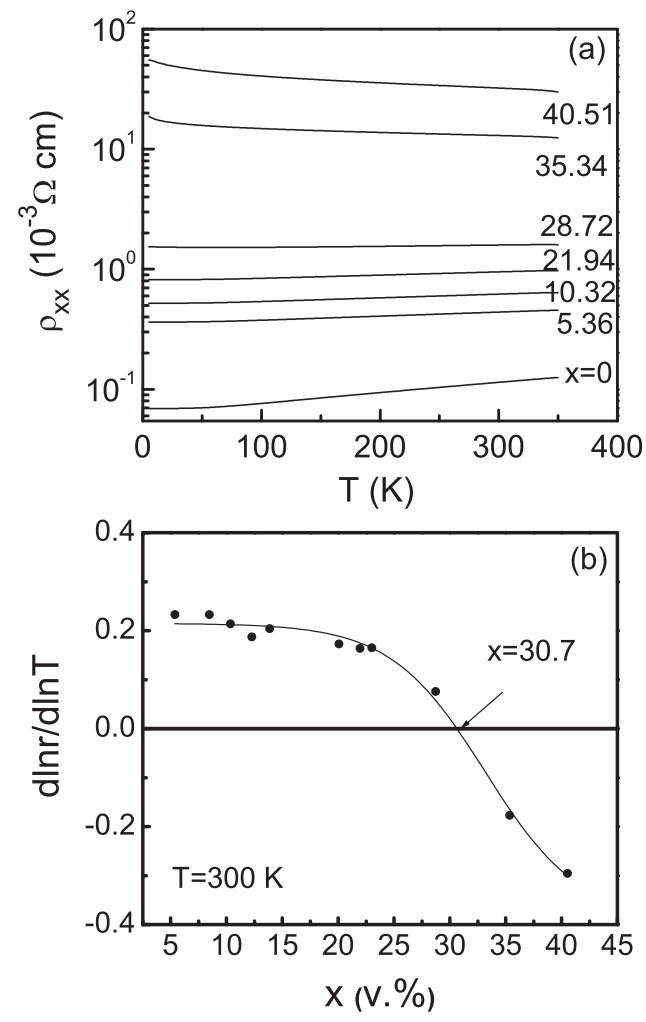

FIG. 3. (a) The temperature-dependent longitudinal resistivity for samples with different $\mathrm{SiO}_{2}$ volume fractions. (b) TCR as a function of the $\mathrm{SiO}_{2}$ volume fraction. The solid line is a guide to the eye.

function of $x$. A sign change at $x \sim 30$ is clearly seen. The positive and negative TCRs normally indicate a metallic behavior and a semiconductor behavior, respectively. For pure $\mathrm{Fe}$, the temperature dependence of resistivity is mainly governed by phonon/magnon scatterings. With the increase of the $\mathrm{SiO}_{2}$ volume fraction, more and bigger insulating clusters formed in the Fe matrix and finally the whole film become insulating, a typical percolation system. ${ }^{18-20}$ When the $\mathrm{SiO}_{2}$ volume fraction approaches the percolation threshold, the material shows very weak temperature dependence. This is because the phonon scatterings become less important in comparison with the scatterings by the insulating impurities and strong structure disorder, which leads to a decreasing TCR [Fig. 3(b)]. Above the percolation threshold, the electrical conduction is dominated by hopping/tunneling processes of electrons. A semiconductorlike behavior appears and the TCR becomes negative. ${ }^{20}$ Normally, the longitudinal resistivity can increase by a few orders in magnitude at the percolation threshold. ${ }^{18,19}$ In this system, the resistivity (at $5 \mathrm{~K}$ ) increased about 800 times as $x$ increased from 0 to 40.51 .

To gain a deeper understanding of the electrical conduction in these materials, we deposited films with similar compositions directly on the TEM $\mathrm{Cu}$ grids that were covered with amorphous carbon film for the TEM experiments. The thickness of the films was less than $100 \mathrm{~nm}$. Shown in Fig. 4 are the TEM images for samples with $x=9$ and 23. At $x$ $=9, \mathrm{SiO}_{2}$ (white strips) exists between the Fe nanocrystals of about $15-20 \mathrm{~nm}$. The thickness of the $\mathrm{SiO}_{2}$ layers was less than $2 \mathrm{~nm}$. Based on the image, one would expect the electrons to be scattered frequently by the $\mathrm{SiO}_{2}$. Therefore, 

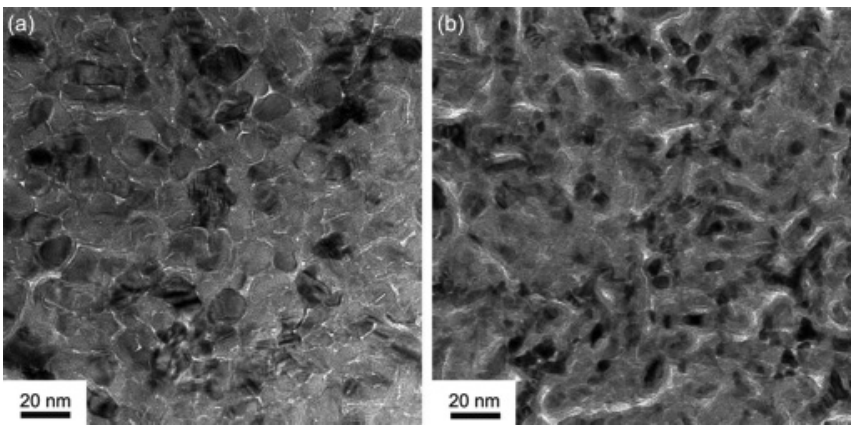

FIG. 4. TEM images for $\mathrm{Fe}_{100-x}\left(\mathrm{SiO}_{2}\right)_{x}$ films: (a) $x=9$ and (b) $x=23$.

the mean-free path (MFP) should be limited to the size of the Fe clusters or shorter, i.e., less than $20 \mathrm{~nm}$, although the MFP in single-crystal Fe films could be as large as $500 \mathrm{~nm}$ at room temperature. ${ }^{23}$ At $x=23$, the feature size of the Fe clusters reduces to about 5-10 $\mathrm{nm}$ and the $\mathrm{SiO}_{2}$ layers become much thicker. Consequently, the MFP in this sample should be much shorter than in the $x=9$ sample. The smaller MFP means more scatterings, which lead to a larger longitudinal resistivity and AHE. This is indeed in line with the composition dependence of resistivity and AHE (Figs. 2 and 3). One should note that the microstructures of the samples shown in Fig. 4 may be slightly different from those in the samples for the AHE measurements because the thicknesses of both sets of samples are quite different.

Now let us understand the opposite trends of Hall resistivity with temperature in the two samples shown in Figs. 1(a) and 1(b). The Hall effect/AHE increases with temperature for the sample with $x=5.36$; whereas the Hall effect/AHE decreases with temperature for the sample with $x=40.51$. The behavior of AHE depends strongly on the behavior of the longitudinal resistivity, based on the scaling laws for intrinsic [Eq. (2)] or extrinsic [Eq. (3)] anomalous Hall effect:

$$
\begin{gathered}
\rho_{x y}^{A} / n \propto \rho_{x x}^{2}, \\
\rho_{x y}^{A} \propto \rho_{x x}^{2} .
\end{gathered}
$$

For the sample with $x=5.36$, the longitudinal resistivity increases monotonically with temperature, which leads to a monotonical increase of the AHE with temperature, Fig. 3(a). However, the longitudinal resistivity decreases monotonically with temperature for $x=40.51$, which certainly leads to a decreasing AHE with temperature.

As discussed previously, by fitting the AHE as a function of magnetic field above the saturation, the ordinary Hall coefficient can be extracted. We therefore calculated the effective carrier density using these OHE coefficients: $\left(R_{O}\right)$ and $n=1 / R_{O} e$, where $e$ is the electronic charge. Shown in Fig. 5 are the calculated effective carrier densities for all the samples. The calculated carrier density for pure $\mathrm{Fe}$ at $300 \mathrm{~K}$ is $16.3 \times 10^{22} / \mathrm{cm}^{3}$, which is in fair agreement with the reported value of $17.0 \times 10^{22} / \mathrm{cm}^{3}{ }^{24}$ This agreement indicates that the carrier density can indeed be obtained from the OHE coefficient in the present study. As expected, with increasing the volume fraction of the insulating phase of $\mathrm{SiO}_{2}$, the effective carrier density decreases monotoni-

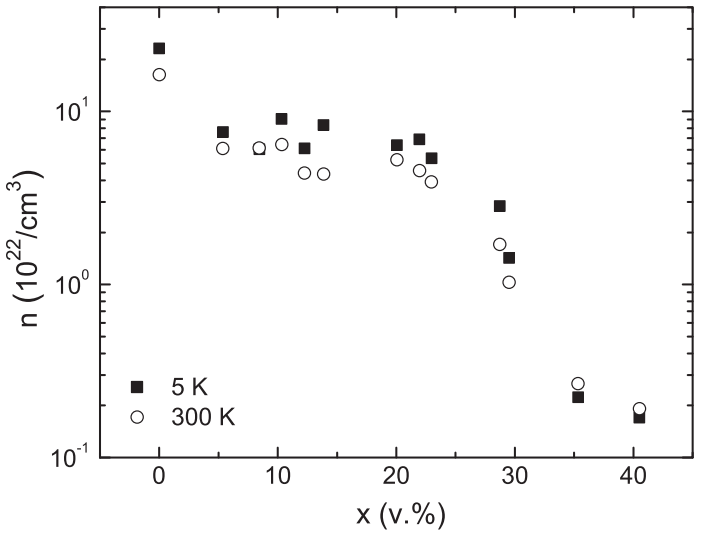

FIG. 5. Effective carrier concentration extracted from the ordinary Hall coefficient versus the $\mathrm{SiO}_{2}$ volume fraction.

cally. Interestingly, the carrier density decreases very sharply when $x$ approaches 30. This dramatic decrease of carrier density can only be understood with the help of percolation theory ${ }^{18}$ and the local quantum interference effect. ${ }^{20}$ Near the percolation, the electrical transport properties are governed by hopping/tunneling and quantum interference processes. $^{18,20}$

Using the effective carrier density (in Fig. 5), AHE resistivity, and longitudinal resistivity, we plotted $\ln \left(\rho_{x y}^{A} / n\right) \sim$ $\ln \left(\rho_{x x}\right)$ in Fig. 6. By fitting the data to a straight line, we obtained the value of $\gamma=2.1 \pm 0.1$ in very broad ranges of variations in longitudinal resistivity (nearly three orders) and AHE resistivity (more than four orders). The perfect scaling between AHE resistivity and longitudinal resistivity is the same as that observed in the ferromagnetic spinel of $\mathrm{CuCr}_{2} \mathrm{Se}_{4-x} \mathrm{Br}_{x}$ by Lee et al., ${ }^{14}$ where this scaling was claimed to be the signature of dissipationless anomalous Hall current, a characteristic of intrinsic AHE. Due to the complex nature of the $\mathrm{Fe}-\mathrm{SiO}_{2}$ granular system (a 3D percolation system), the $\mathrm{N}$-L theory may not be applied directly to interpret the data. However, this scaling should be a very interesting observation in the magnetic granular/magnetic percolation systems. It seems that the anomalous Hall conductivity is independent

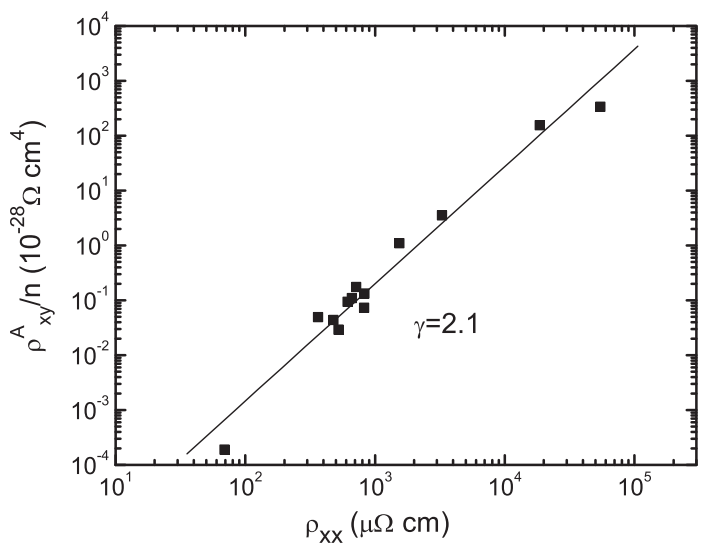

FIG. 6. The normalized anomalous Hall resistivity obtained at $5 \mathrm{~K}$ versus the longitudinal resistivity in a log-log scale for all of the samples. The line is a linear fit of the data, with a slope $\gamma=2.1 \pm$ 0.1 . 


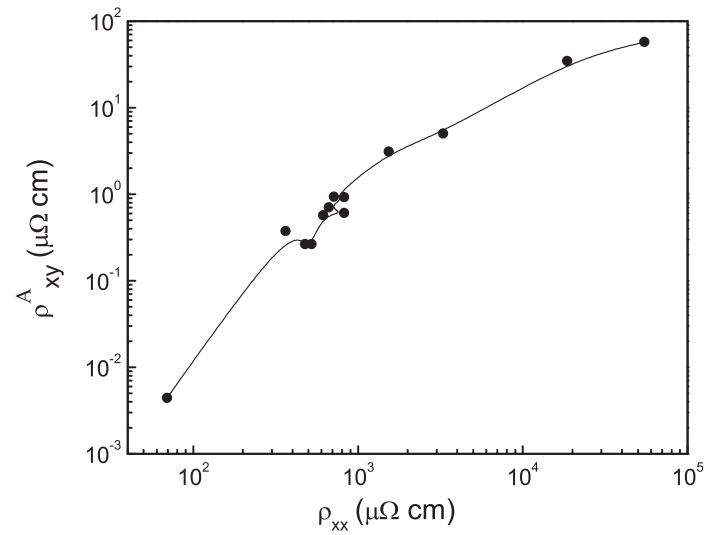

FIG. 7. AHE resistivity (5 K) as a function of longitudinal resistivity in a log-log scale. The solid line is a guide to the eye.

of the scattering when a good ferromagnetic metal transforms into a percolated system.

In the $\mathrm{Fe}-\mathrm{SiO}_{2}$ granular system, electrical transports are determined by transport within $\mathrm{Fe}$ clusters and hopping/tunneling between the clusters. All of the iron oxide phases $\left(\mathrm{FeO}, \mathrm{Fe}_{2} \mathrm{O}_{3}\right)$, if existent in the samples, are quite insulating. The oxide phases may exist as $\mathrm{Fe} / \mathrm{FeO}$ core/shell clusters, ${ }^{25}$ interfaces between the Fe clusters, or embedment in the $\mathrm{SiO}_{2}$ matrix. Based on the low-temperature magnetization data [Figs. 1(c) and 1(d)], it is known that the oxide phase indeed formed because the saturation magnetization is lower than the theoretical value. This may also explain why the percolation threshold is about $30 \% \mathrm{SiO}_{2}$, being lower than $50 \%$ as previously reported. ${ }^{12}$ However, the existence of iron oxide in the films does not affect the interpretation of the observation here because the iron oxide played the same role as the insulating $\mathrm{SiO}_{2}$.

One may argue that the side-jump mechanism (the extrinsic AHE) found by Berger ${ }^{4}$ also leads to a square dependence of the AHE resistivity on the longitudinal resistivity, i.e., $\rho_{x y}^{A} \propto \rho_{x x}^{2}$. If the carrier density $(n)$ is constant or very weakly depends on the sample composition, the behavior of Fig. 6 may also be ascribed to the side-jump mechanism. In order to clarify this issue, we plotted the AHE resistivity as a function of longitudinal resistivity in a log-log scale in Fig. 7. It is clearly seen that the data cannot be fitted to a straight line, i.e., the data cannot be scaled by square dependence, $\rho_{x y}^{A} \propto \rho_{x x}^{2}$. Actually, the data cannot be fitted to any power law, $\rho_{x y}^{A} \propto \rho_{x x}^{n}$. Therefore, the data cannot be described by any extrinsic AHE models, i.e., neither by the side-jump model $\left(\rho_{x y}^{A} \propto \rho_{x x}^{2}\right)$ nor by the skew scattering model $\left(\rho_{x y}^{A} \propto \rho_{x x}\right)$. Since both the SS and SJ models were based on the scattering of electrons by the impurities/imperfections, the Hall conductivity should be closely related to the scattering strength and frequency. However, the scaling $\rho_{x y}^{A} / n \propto \rho_{x x}^{2}$ might suggest that the $\mathrm{AHE}$ in the $\mathrm{Fe}-\mathrm{SiO}_{2}$ system is scattering independent.

\section{CONCLUSION}

We investigated the composition- and temperaturedependent Hall resistivity and longitudinal resistivity in the $\mathrm{Fe}_{100-x}\left(\mathrm{SiO}_{2}\right)_{x}$ granular films. The key finding is the scaling between the normalized anomalous Hall resistivity by carrier density and the longitudinal resistivity scaling $\left(\rho_{x y}^{A} / n \propto \rho_{x x}^{2}\right)$. This scaling might suggest a scattering-independent anomalous Hall conductivity in Fe. However, a full understanding of the AHE in the percolation systems requires experimental studies of different material systems.

\section{ACKNOWLEDGMENTS}

The work described in this paper was supported partially by grants from the Research Grants Council of the Hong Kong Special Administrative Region (Project No. 604407) and the National Science Foundation of China (Contracts No. 50729101 and No. 50525101).
*xixiang.Zhang@kaust.edu.sa

${ }^{1}$ L. Berger and G. Bergmann, in The Hall Effect and Its Applications, edited by C. L. Chien and C. R. Westgate (Plenum, New York, 1979), p. 55, and references therein.

${ }^{2}$ R. Karplus and J. M. Luttinger, Phys. Rev. 95, 1154 (1954);

J. M. Luttinger, ibid. 112, 739 (1958).

${ }^{3}$ J. Smit, Physica 21, 877 (1955); 24, 39 (1958).

${ }^{4}$ L. Berger, Phys. Rev. B 2, 4559 (1970).

${ }^{5}$ H. Leribaux, Phys. Rev. 150, 384 (1966).

${ }^{6}$ Y. G. Yao, L. Kleinman, A. H. MacDonald, J. Sinova, T. Jungwirth, D. S. Wang, E. G. Wang, and Q. Niu, Phys. Rev. Lett. 92, 037204 (2004).

${ }^{7}$ T. Jungwirth, Q. Niu, and A. H. MacDonald, Phys. Rev. Lett. 88, (2002) 207208.

${ }^{8}$ Z. Fang, N. Nagaosa, K. S. Takahashi, A. Asamitsu, R. Mathieu,

T. Ogasawara, H. Yamada, M. Kawasaki, Y. Tokura, and

K. Terakura, Science 302, 92 (2003); R. Mathieu, A. Asamitsu,

H. Yamada, K. S. Takahashi, M. Kawasaki, Z. Fang, N. Nagaosa, and Y. Tokura, Phys. Rev. Lett. 93, 016602 (2004).
${ }^{9}$ J. W. Ye, Y. B. Kim, A. J. Millis, B. I. Shraiman, P. Majumdar, and

Z. Tesanovic, Phys. Rev. Lett. 83, 3737 (1999).

${ }^{10}$ G. Sundaram and Q. Niu, Phys. Rev. B 59, 14915 (1999).

${ }^{11}$ A. B. Pakhomov, X. Yan, and B. Zhao, Appl. Phys. Lett. 67, 3497 (1995).

${ }^{12}$ B. Zhao and X. Yan, J. Appl. Phys. 81, 4290 (1997).

${ }^{13}$ B. A. Aronzon, D. Y. Kovalev, A. N. Largar'kov, E. Z. Meilikhov, V. V. Ryl'kov, M. A. Sedova, N. Negre, and M. Goiran, JETP Lett. 70, 90 (1999).

${ }^{14}$ W.L. Lee, S. Watauchi, V. L. Miller, R. J. Cava, and N. P. Ong, Science 303, 1647 (2004)

${ }^{15} \mathrm{P}$. Nozières and C. Lewiner, J. Phys. (France) 34, 901 (1973).

${ }^{16}$ K. Miyatani, K. Minematsu, Y. Wada, F. Okomoto, K. Kato, and P. K. Baltzer, J. Phys. Chem. Solids 32, 1429 (1971).

${ }^{17}$ J. B. Goodenough, J. Phys. Chem. Solids 30, 261(1969).

${ }^{18}$ D. J. Bergman and D. Stroud, Solid State Phys. 46, 149 (1992); D. Stauffer and A. Aharony, Introduction to Percolation Theory (Taylor \& Francis, Bristol, 1994). 
${ }^{19}$ U. Dai, A. Palevski, and G. Deutscher, Phys. Rev. B 36, 790 (1987); E. K. Sichel and J. I. Gittleman, Solid State Commun. 42, 75 (1982).

${ }^{20}$ X. X. Zhang, C. Wan, H. Liu, Z. Q. Li, P. Sheng, and J. J. Lin, Phys. Rev. Lett. 86, 5562 (2001); C. C. Wan and P. Sheng, Phys. Rev. B 66, 075309 (2002).

${ }^{21}$ N. A. Sinitsyn, J. Phys. Condens. Matter 20, 023201 (2008).
${ }^{22}$ N. Nagaosa, J. Sinova, S. Onoda, A. H. MacDonald, and N. P. Ong, Rev. Mod. Phys. 82, 1539 (2010).

${ }^{23}$ C. Martinez-Boubeta, Ll. Balcells, and A. Cebollada, Appl. Phys. Lett. 88, 132511(2006).

${ }^{24}$ N. W. Ashcroft and N. D. Mermin, Solid State Physics (Thomas Learning, Inc, London, 1976), p. 5.

${ }^{25}$ K. K. Fung, B. X. Qin, and X. X. Zhang, Mater. Sci. Eng. A 286, 135 (2000). 